\title{
Cytotoxicity of Brucella smooth strains for macrophages is mediated by increased secretion of the type IV secretion system
}

Correspondence

Zeliang Chen

zeliangchen@yahoo.com

Kehe Huang

khhuang@njau.edu.cn

Received 6 May 2009

Revised 15 July 2009

Accepted 16 July 2009

\author{
Zhijun Zhong, ${ }^{1,2}+$ Yufei Wang, ${ }^{2}$ † Feng Qiao, ${ }^{2} \dagger$ Zhoujia Wang, ${ }^{2}$ \\ Xinying Du, ${ }^{2}$ Jie Xu, ${ }^{2}$ Jin Zhao, ${ }^{2}$ Oing Ou, ${ }^{2}$ Shicun Dong, ${ }^{2}$ Yansong Sun, ${ }^{2}$ \\ Liuyu Huang, ${ }^{2}$ Kehe Huang ${ }^{1}$ and Zeliang Chen $^{2}$ \\ ${ }^{1}$ College of Veterinary Medicine, Nanjing Agricultural University, Nanjing 210095, Jiangsu Province, \\ PR China \\ ${ }^{2}$ Institute of Disease Control and Prevention, Academy of Military Medical Science, Beijing 100071, \\ PR China
}

\begin{abstract}
Some Brucella rough mutants cause cytotoxicity that resembles oncosis and necrosis in macrophages. This cytotoxicity requires the type IV secretion system (T4SS). In rough mutants, the cell-surface $\mathrm{O}$ antigen is shortened and the T4SS structure is thus exposed on the surface. Cytotoxicity effector proteins can therefore be more easily secreted. This enhanced secretion of effector proteins might cause the increased levels of cytotoxicity observed. However, whether this cytotoxicity is unique to the rough mutant and is mediated by overexpression of the T4SS has not been definitively determined. To test this, in the present study, a virB inactivation mutant (BM $\Delta$ virB) and an overexpression strain (BM-VIR) of a smooth Brucella melitensis strain (BM) were constructed and their cytotoxicity for macrophages and intracellular survival capability were analysed and compared. Cytotoxicity was detected in macrophages infected with higher concentrations of strains BM or BM-VIR, but not in those infected with $\mathrm{BM} \Delta \mathrm{virB}$. The quorum sensing signal molecule $N$-dodecanoyl-DL-homoserine lactone $\left(\mathrm{C}_{12}-\mathrm{HSL}\right)$, a molecule that can inhibit expression of virB, inhibited the cytotoxicity of BM and BM-VIR, but not of BM $\Delta$ virB. These results indicated that overexpression of virB is responsible for Brucella cytotoxicity in macrophages. Transcription analysis showed that virB is regulated in a cell-density-dependent manner both in in vitro culture and during macrophage infection. When compared with BM, BMVIR showed a reduced survival capacity in macrophages and mice, but both strains demonstrated similar resistance to in vitro stress conditions designed to simulate intracellular environments.

Taken together, the cytotoxicity of Brucella for macrophages is probably mediated by increased secretion of effector proteins that results from overexpression of virB or an increase in the number of bacterial cells. The observation that both inactivation and overexpression of vir $B$ are detrimental for Brucella intracellular survival also indicated that the expression of virB is tightly regulated in a cell-density-dependent manner.
\end{abstract}

\section{INTRODUCTION}

Brucella spp. are Gram-negative intracellular pathogens, belonging to the class Alphaproteobacteria, which live in close association with eukaryotic hosts. Bacteria of the genus Brucella are the aetiological agents of brucellosis, a worldwide zoonosis which affects a broad range of mammals and causes great economic losses. According to conventional taxonomy, three Brucella species, Brucella

†These authors contributed equally to this work.

Abbreviations: $\mathrm{C}_{12}-\mathrm{HSL}, \mathrm{N}$-dodecanoyl-DL-homoserine lactone; LDH, lactate dehydrogenase; T4SS, type IV secretion system. melitensis, Brucella abortus and Brucella suis, can infect humans, causing a chronic, debilitating disease with severe, and sometimes fatal outcomes.

Brucella is well adapted to an intracellular lifestyle, being able to invade and survive in both phagocytic and nonphagocytic cells. Internalization of Brucella occurs through lipid-raft-mediated macropinocytosis (Kim et al., 2002; Watarai et al., 2002). After being taken up into the phagosome, Brucella is packaged into vacuoles called Brucella-containing vacuoles, which move in host cells by a specific intracellular trafficking mechanism that avoids fusion with lysosomes. Brucella-containing vacuoles fuse 
with the endoplasmic reticulum membrane and replicate in vesicles associated with the endoplasmic reticulum (Celli et al., 2003, 2005; Gorvel \& Moreno, 2002; Pizarro-Cerda et al., 1998; Starr et al., 2008). The type IV secretion system (T4SS), encoded by the virB operon, plays a central role in Brucella intracellular survival and replication (Foulongne et al., 2000; Hong et al., 2000; Lestrate et al., 2000; O'Callaghan et al., 1999). virB mutants are unable to persist within host cells and are therefore unable to cause chronic infection in mice (Alvarez-Martinez et al., 2001; Boschiroli et al., 2002a, b; Comerci et al., 2001; O'Callaghan et al., 1999; Sieira et al., 2000).

The function of the T4SS in bacterial intracellular survival has been shown to be mediated by effector proteins that are secreted into host cells. Other studies have suggested that the virB operon of Brucella mediates secretion of unknown effectors which are required for establishment of an intracellular compartment favourable for Brucella survival and replication (Boschiroli et al., 2002b). This is supported by data from co-infection experiments which show that wild-type strains of $B$. abortus or B. suis are able to rescue virB mutants in macrophages, and that this rescue requires bacterial protein synthesis (Nijskens et al., 2008). de Jong et al. (2008) have recently demonstrated that two members of the $v j b R$ regulon, $\mathrm{VceA}$ and $\mathrm{VceC}$, are translocated into macrophages by the T4SS, providing the first direct evidence for the secretion of effector proteins by the Brucella T4SS.

The cytotoxicity of Brucella for macrophages was observed more than 40 years ago (Freeman \& Rumack, 1964). It has been shown more recently that rough mutants are more cytotoxic to macrophages than smooth strains (Pei \& Ficht, 2004), and these authors predicted that rough mutants secrete more toxin molecules into host cells. The cytotoxicity of rough mutants for macrophages resembles oncosis and necrosis, but not apoptosis, and requires bacterial protein synthesis (Pei \& Ficht, 2004; Pei et al., 2006). It is presumed that an $\mathrm{O}$ antigen expressed on the bacterial cell surface of smooth strains may affect effector secretion of T4SS. This has been observed for type III secretion systems in Shigella and Yersinia (Mota et al., 2005; West et al., 2005). In Shigella, shortening of the lipopolysaccharide molecule by $\mathrm{O}$-antigen glucosylation enhances the secretion of effector proteins and the function of the type III secretion system. A minimal 'needle' length is required for efficient functioning of the Yersinia enterocolitica type III secretion system. In smooth Brucella strains, the T4SS apparatus might be partially shielded by the $\mathrm{O}$ antigen, and therefore the effector proteins are secreted in a tightly controlled way. In contrast, for the rough Brucella strains, the $\mathrm{O}$ antigen is lacking or is shortened, the T4SS structure is more exposed on the surface and the effector proteins can be easily secreted. The enhanced secretion of effector proteins might cause the increased cell death (Pei \& Ficht, 2004; Pei et al., 2006, 2008).

Although several reports have shown that the cytotoxicity of the rough mutant is mediated by the increased secretion of effector proteins via the T4SS, there is no direct evidence to show that this cytotoxicity is a unique rough strain phenotype mediated by the T4SS. Based on previous studies, it can be predicted that the T4SS is tightly regulated in smooth strains during its intracellular survival in host cells. To test this in the present study, a virB inactivation mutant $(\mathrm{BM} \Delta \mathrm{virB})$ and a $\operatorname{vir} B$ overexpression strain (BM-VIR) of a smooth strain of B. melitensis were constructed. Their cytotoxicity for macrophages and transcription of virB was analysed and compared. The results showed that overexpression of virB in B. melitensis increased the cytotoxicity of smooth Brucella for macrophages, and in contrast, inactivation of virB prevented the cytotoxic effects. Transcription of virB is growth-phasedependent in vitro and infection-stage-dependent during macrophage infection. These results indicate that the cytotoxicity of smooth strains is mediated by $\operatorname{vir} B$, and that expression of $v i r B$ in smooth strains is tightly regulated during macrophage infection.

\section{METHODS}

Bacterial strains, plasmids, primers and reagents. The virB inactivation mutant $\mathrm{BM} \Delta \mathrm{virB}$ and $\operatorname{vir} B$ overexpression strain BM-VIR were constructed as described below. Brucella strains were grown in tryptic soy broth (TSB) or on tryptic soy agar (TSA). Escherichia coli $\mathrm{DH} 5 \alpha$ was grown in Luria-Bertani (LB) medium. When necessary, ampicillin (Ap; $100 \mu \mathrm{g} \mathrm{ml}^{-1}$ ) and kanamycin $\left(\mathrm{Km} ; 50 \mu \mathrm{g} \mathrm{ml}{ }^{-1}\right.$ ) were added to the cultures. Plasmid pBBR1MCS-4, a broad-host-range plasmid capable of replicating in Brucella, was provided by Professor Kenneth M. Peterson (Kovach et al., 1995). The primers used in this study are listed in Table 1. The CytoTox 96 non-radioactive cytotoxicity assay kit was purchased from Promega. The autoinducer $\mathrm{N}$-dodecanoyl-DL-homoserine lactone $\left(\mathrm{C}_{12}\right.$-HSL) was obtained from Fluka.

Construction of the virB inactivation mutant BM $\Delta$ virB. $B M \Delta$ virB was constructed from B. melitensis 55009 (BM), a strain derived from $16 \mathrm{M}$. The $1.7 \mathrm{~kb} s a c B$ gene was released from pKOBEG-SacB by NdeI and cloned into the NdeI site of pUC19 to give pUC19-SacB. This allowed positive selection of double cross-over events by selection using sucrose resistance $\left(\mathrm{Suc}^{\mathrm{R}}\right)$. A $504 \mathrm{bp}$ region just upstream of virB1 was deleted as follows. The $\mathrm{N}$-terminal fragment was amplified with primers IVB-N-F and IVB-N-R, and the C-terminal fragment with IVB-C-F and IVB-C-R. The two PCR products were purified, equally mixed, and then amplified with primers IVB-N-F and IVB-C$\mathrm{R}$ to generate IVB that was cloned into $\mathrm{pUC19-SacB}$ to generate pUCSacB-IVB. Competent BM was transformed with pUCSacB-IVB and the $\operatorname{vir} B$ mutant $B M \Delta v i r B$ was isolated by its $A^{S}{ }^{S} c^{R}$ phenotype. The unmarked deletion mutant was further confirmed by PCR and DNA sequencing.

Construction of the virB overexpression strain BM-VIR. The virB overexpression strain was constructed as follows. A $1087 \mathrm{bp}$ fragment located downstream of the virB operon was amplified with IVGT-F and IVGT-R and cloned into pUC19 to generate the targeting plasmid pUC19-IVT. This plasmid was then transformed into competent BM, resulting in targeted strain BM-IVT. Genomic DNA was extracted from BM-IVT and digested with EcoT22I, and then purified, selfligated and transformed into DH5 $\alpha$ to rescue plasmid pUC19-IVGT. The rescued $v i r B$ operon in pUC19-IVGT was confirmed by PCR with primers for virB2, virB5 and virB8, and the PCR products were sequenced for sequence confirmation. The two termini of the rescued 
Table 1. Primers used in this study

\begin{tabular}{|ll|}
\hline Primer & \multicolumn{1}{c|}{ Sequence $\left(\mathbf{5}^{\prime} \mathbf{}^{\prime}{ }^{\prime}\right)$} \\
\hline IVB-N-F & CTGCGAAGCTTGCAAATTCCCGTCCGGTTCG \\
IVB-N-R & GAGGACAAGGAATGGCACCACGACGCAGGACGGAAAGGAC \\
IVB-C-F & GTCCTTTCCGTCCTGCGTCGTGGTGCCATTCCTTGTCCTC \\
IVB-C-R & CGACCGGAATTCGAAGCCGCCCGTAAAGTTGC \\
IVGT-F & ACGTCGGATCCGAATTCACAGGCATTATCCGCTCGTC \\
IVGT-R & AGTCGAAGCTTTCTAGAAGCATAGCCAGTAGGTCCAG \\
virB2-F & ATGAAAACCGCTTCCCCCAGC \\
virB2-R & CCTAAGCAGGTAAGAGGC \\
virB5-F & ATGAAGAAGATAATTCTCAGC \\
virB5-R & ATAGGCGGCTTCCAGTGC \\
virB8-F & ATGTTTGGACGCAAACAATCTC \\
virB8-R & TTGCACCACTCCCATTTCTG \\
virB1-RT-F & AAGCAATCACGACAGCACAG \\
virB1-RT-R & CGGCGTAGTAACAGGAGAATG \\
virB8-RT-F & GGGCTTTCGGCACCATTAC \\
virB8-RT-R & AGCGTGTACCAGTCGTAGG \\
16SRNA-RT-F & CACTGGACCATTACTGACGC \\
16SRNA-RT-R & ACTAAGGGCGAGGGTTGC \\
\hline
\end{tabular}

fragment were confirmed by sequencing with sequencing primers of pUC19. The virB operon was released from pUC19 by digestion with $K p n \mathrm{I}$ and PstI, and subcloned into pBBR1MCS-4 to generate pBBR1IVGT, which was electroporated into strain $\mathrm{BM}$, resulting in the overexpression strain BM-VIR.

Reverse transcription PCR (RT-PCR). For transcriptional analysis of virB, strains BM, BM $\Delta$ virB and BM-VIR were cultured in TSB to exponential phase $\left(\mathrm{OD}_{600}=1.0\right)$ and then subjected to RNA isolation. Total RNA was isolated with Trizol agent (Invitrogen) as recommended by the manufacturer. RNA samples were then treated with DNase I (Promega) to remove contaminating genomic DNA. RNA quantity and quality were analysed by using an ND-1000 Spectrophotometer (Nanodrop Technologies). cDNA was generated from total RNA by using a random hexamer primer, following the protocol of Superscript II reverse transcriptase (Invitrogen). 16S rRNA, which is constantly transcribed in bacteria, was chosen as an internal control. Different cDNA samples were amplified with primers for $16 \mathrm{~S}$ rRNA. The cDNA samples were normalized by differential dilutions according to the quantity of $16 \mathrm{~S}$ rRNA products. Then, virB1 and virB8 were amplified from normalized cDNA samples with specific primers. The PCR products were analysed on a $1 \%$ agarose gel and visualized by ethidium bromide staining.

Real-time quantitative RT-PCR (qRT-PCR). The primers used for qRT-PCR are listed in Table 1 . Samples were run in triplicate and amplified in a $20 \mu \mathrm{l}$ reaction containing $10 \mu \mathrm{l} 2 \times$ SYBR Green I Master Mix (TaKaRa), $100 \mathrm{nM}$ forward/reverse primer and $1 \mu \mathrm{l}$ cDNA sample. Moreover, no-template controls were used as recommended. qRT-PCR was performed by using the Roche LightCycler 2.0 system. The reaction conditions were as follows: denaturation at $95{ }^{\circ} \mathrm{C}$ for $15 \mathrm{~s}$, and then 50 cycles of amplification $\left(95{ }^{\circ} \mathrm{C}\right.$ for $5 \mathrm{~s}$, and $60{ }^{\circ} \mathrm{C}$ for $20 \mathrm{~s}$ ). The relative transcriptional level was determined as described by Livak \& Schmittgen (2001): relative fold change (treatment/control) $=2^{-\Delta \Delta C_{\mathrm{T}}}$, where $\Delta C_{\mathrm{T}}$ (gene of interest $)=C_{\mathrm{T}}$ (gene of interest $)-C_{\mathrm{T}}$ (reference gene of same sample), and $\Delta \Delta C_{\mathrm{T}}$ (gene of interest) $=\Delta C_{\mathrm{T}}$ (treatment) $-\Delta C_{\mathrm{T}}$ (control). The $16 \mathrm{~S}$ rRNA gene, the expression of which is relatively constant in bacteria, was used as the reference gene, and bacteria grown in TSB7.0 were used as control.
Macrophage infection and intracellular survival assay. Murine macrophage-like J774A.1 cells were seeded in 24-well plates and infected with B. melitensis at an m.o.i. of 200 , as described by Pei \& Ficht (2004). To synchronize the infection, the infected plates were centrifuged at $200 \mathrm{~g}$ for $5 \mathrm{~min}$ at room temperature. Following 60 min incubation at $37{ }^{\circ} \mathrm{C}$ in an atmosphere containing $5 \%(\mathrm{v} / \mathrm{v})$ $\mathrm{CO}_{2}$, the cells were washed three times with PBS to remove extracellular bacteria. To assess intracellular growth of the bacteria, the cells were incubated at $37{ }^{\circ} \mathrm{C}$ in fresh DMEM supplemented with $100 \mu \mathrm{g} \mathrm{Ap} \mathrm{ml}^{-1}$ plus $50 \mu \mathrm{g} \mathrm{Km} \mathrm{ml}{ }^{-1}$ (to kill extracellular bacteria) for $1 \mathrm{~h}$, then the concentration of antibiotics was reduced to 50 or $25 \mu \mathrm{g} \mathrm{ml}^{-1}$, respectively (time zero). At different time points postinfection (p.i.), the supernatant was discarded and cells were lysed with $0.1 \%(\mathrm{v} / \mathrm{v})$ Triton X-100. The number of c.f.u. was obtained by plating serial dilutions of the lysates on TSA plates. All survival assays were performed in triplicate wells, and the results represent the means from at least three separate experiments. For transcription analysis during macrophage infection, at different time points p.i., total RNA was isolated with Trizol agent. RT-PCR and qRT-PCR analyses were carried out as described above. RNA of uninfected J774A.1 cells was isolated and used as a negative control.

Macrophage cytotoxicity assay. Cells cultured in 24-well plates were infected with B. melitensis strains at an m.o.i. of 20, 200 or 2000 as described above. The culture supernatant was collected at various time points p.i., and the level of lactate dehydrogenase (LDH) was determined by the Promega CytoTox 96 non-radioactive cytotoxicity assay, according to the manufacturer's instructions with minor modifications (Pei \& Ficht, 2004). Cytopathic cell death is expressed as a percentage of maximum $\mathrm{LDH}$ release, i.e. $100 \times\left(\mathrm{OD}_{490}\right.$ of infected cells $-\mathrm{OD}_{490}$ of uninfected cells $) /\left(\mathrm{OD}_{490}\right.$ of lysed uninfected cells $-\mathrm{OD}_{490}$ of uninfected cells). The maximum release was determined following dissolution of cell monolayers using $1 \%$ Triton X-100. The assays were performed in triplicate wells, and the data represent the mean \pm sD from at least three separate experiments.

Autoinducer $\mathrm{C}_{12}$-HSL treatment of Brucella-infected cells. Macrophages cultured in 24 -well plates were infected with $B$. melitensis strains at an m.o.i. of 200 as described above. The infected cells were treated with fresh media containing various concentrations 
of $\mathrm{C}_{12}$-HSL at $1 \mathrm{~h}$ p.i., and cytotoxicity was determined at $24 \mathrm{~h}$ p.i. using the $\mathrm{LDH}$ release assay.

Mouse virulence assay. Female 6-week-old BALB/c mice were inoculated intraperitoneally with $0.2 \mathrm{ml}$ PBS containing approximately $5 \times 10^{7}$ c.f.u. of $B$. melitensis. Infected mice were held in microisolator cages in biosafety level 3 facilities. Bacterial counts in the spleen or liver were determined for five mice of each group at 3, 5, 7, 14 and 28 days p.i. Mice were euthanized by $\mathrm{CO}_{2}$ asphyxiation and the spleens and livers were collected aseptically. Spleens and livers from individual animals were homogenized with $1 \mathrm{ml} 0.1 \%(\mathrm{v} / \mathrm{v})$ Triton X-100 in PBS, 10-fold serially diluted, and plated on TSA. Plates were incubated at $37{ }^{\circ} \mathrm{C}$ and the c.f.u. was counted 3 days later. The c.f.u. per spleen or per liver was transformed exponentially to normalize the distribution. Results were expressed as the mean \pm SD. All animal experiments were approved by the Academy of Military Medical Science Laboratory Animal Care and Use Committee and conducted in accordance with institutional guidelines.

In vitro environmental stress. $B$. melitensis strains were grown in TSB to the early exponential phase $\left(\mathrm{OD}_{600}=0.6\right)$ at $37^{\circ} \mathrm{C}$, and then subjected to different in vitro stress treatments. The in vitro stress conditions were designed to simulate intracellular environments and were chosen as described by Teixeira-Gomes et al. (2000) with minor modifications. To determine the effect of oxidative stress, bacteria grown to exponential phase were incubated in $440 \mathrm{mM} \mathrm{H}_{2} \mathrm{O}_{2}$ for $30 \mathrm{~min}$. For low pH stress, bacteria were incubated at $37{ }^{\circ} \mathrm{C}$ in TSB medium at $\mathrm{pH} 2.5$ for $5 \mathrm{~min}$. For high-temperature stress, bacterial cultures were transferred to pre-warmed $50{ }^{\circ} \mathrm{C}$ tubes and incubated at $50{ }^{\circ} \mathrm{C}$ for $60 \mathrm{~min}$. After the treatment, cells were diluted and plated on TSB agar to determine the number of viable bacteria. All the results represent the means from at least three separate experiments. The relative transcription of virB under these conditions was quantified by qRT-PCR.

Growth curve and transcription analysis of virB in different growth phases. BM and BM-VIR were cultured in TSB for $24 \mathrm{~h}$, and then diluted with TSB to an $\mathrm{OD}_{600}$ of 0.05 and cultured in a rotary shaker (250 r.p.m.) at $37{ }^{\circ} \mathrm{C}$. Aliquots of cultures were taken at intervals of $2 \mathrm{~h}$ and cell density $\left(\mathrm{OD}_{600}\right)$ was recorded. Transcription of $\operatorname{virB}$ at different growth stages was analysed by qRT-PCR.

Statistical analysis. Statistical significance was determined using Student's $t$-test; a $P$-value of $<0.05$ was considered significant, and a $P$-value of $<0.01$ was considered greatly significant.

\section{RESULTS}

\section{Construction of the virB inactivation strain $B M \Delta v i r B$ and overexpression strain BM-VIR}

Eleven sequential ORFs encoding type IV secretion machinery make up the $\operatorname{vir} B$ operon, making $\operatorname{vir} B$ inactivation possible by promoter deletion. To construct an unmarked virB deletion mutant, the counter-selection gene $s a c B$ was first cloned into pUC19 to give pUC19-sacB. This plasmid was then used to construct the virB mutant as described in Methods. The unmarked deletion mutant $\mathrm{BM} \Delta \mathrm{virB}$, which resulted from two rounds of cross-over, was obtained by its $\mathrm{Ap}^{\mathrm{S}} \mathrm{Suc}^{\mathrm{R}}$ phenotype (data not shown). To construct the virB overexpression strain, $\operatorname{vir} B$ was first cloned by plasmid rescue as described in Methods. PCR and DNA sequencing of the internal virB genes and the two termini showed that $\operatorname{vir} B$ was correctly cloned (data not shown). virB was then subcloned into pBBR1MCS-4 to yield complementary plasmid pBBR-IVGT, and confirmed by PCR and sequencing. This plasmid was transformed into strain $\mathrm{BM}$ and the virB overexpression strain BM-VIR was generated. To confirm the two strains, transcription of virB genes was quantified by RT-PCR. As shown in Fig. 1, the abundance of virB1 and virB8 in strain BM-VIR was higher than that in strain $\mathrm{BM}$, and no transcripts for these genes were detected in strain $\mathrm{BM} \Delta \mathrm{virB}$. This indicated that virB genes were overexpressed in BM-VIR and inactivated in $\mathrm{BM} \Delta$ virB.

\section{Smooth strain BM is cytotoxic for macrophages when infected at a high m.o.i.}

Cytotoxicity is classically evaluated by the quantification of plasma membrane damage. LDH is a stable cytosolic enzyme, present in all cell types, and rapidly released into the cell culture medium upon damage of the plasma membrane. $\mathrm{LDH}$, therefore, is the most widely used marker in cytotoxicity studies. To test whether the smooth strain $\mathrm{BM}$ is cytotoxic for macrophages, J774A.1 cells were infected with BM at m.o.i.s of 20, 200 and 2000, and LDH release was analysed. As shown in Fig. 2(a), at 12 h p.i., for macrophages infected with $\mathrm{BM}$ at an m.o.i. of 20 , only $3.29+1.11 \%$ LDH was released; however, for those infected at m.o.i.s of 200 and 2000, LDH release increased to $33.53 \pm 1.67$ and $60.30 \pm 2.36 \%$, respectively $(P<0.01)$. This indicated that, at higher m.o.i., more LDH was released. The LDH release at $24 \mathrm{~h}$ was higher than that at $12 \mathrm{~h}$ p.i. for all m.o.i.s. However, when the infected cells were cultured for $48 \mathrm{~h}$ p.i., all macrophages showed high levels of $\mathrm{LDH}$ release and no great differences between those infected at different m.o.i.s were observed $(P>0.05)$, implying that at this time point, most of the infected cells

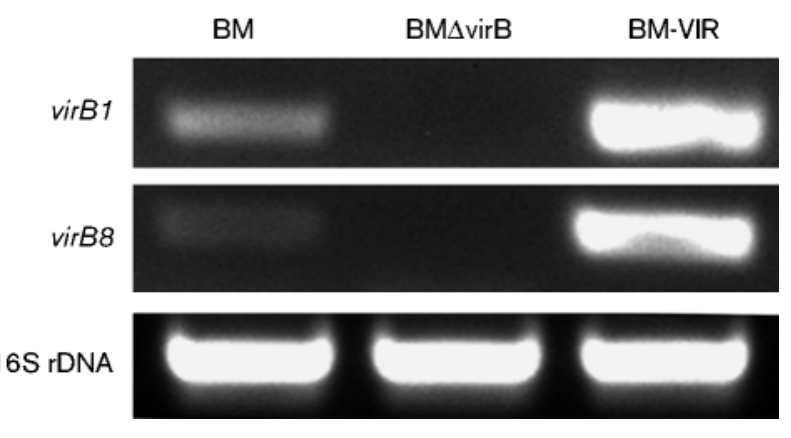

Fig. 1. RT-PCR verification of virB transcription in strains $B M$, $\mathrm{BM} \Delta$ virB and BM-VIR. RNA was isolated from strains $B M$, $\mathrm{BM} \Delta \mathrm{virB}$ and $\mathrm{BM}-\mathrm{VIR}$ and then reverse-transcribed into cDNA. cDNA samples of the three strains were normalized by the transcription level of $16 \mathrm{~S}$ rRNA. Transcription of virB genes (virB1 and virB8) was detected in $\mathrm{BM}$ and $\mathrm{BM}-\mathrm{VIR}$, but not in $\mathrm{BM} \Delta$ virB. The transcription level of virB genes of BM-VIR was much higher than in BM. 

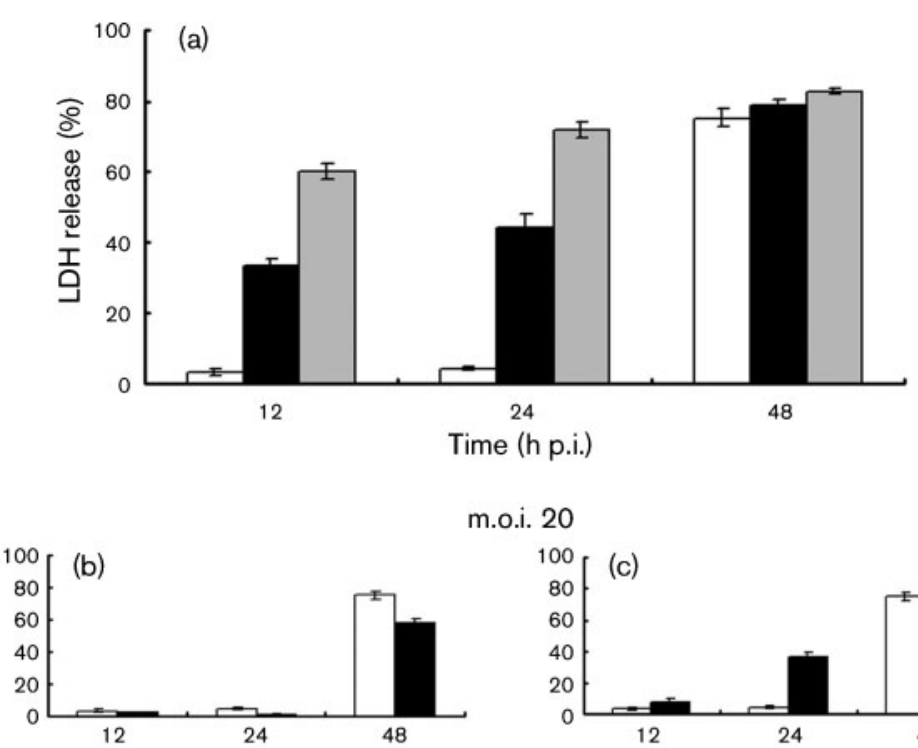

m.o.i. 20
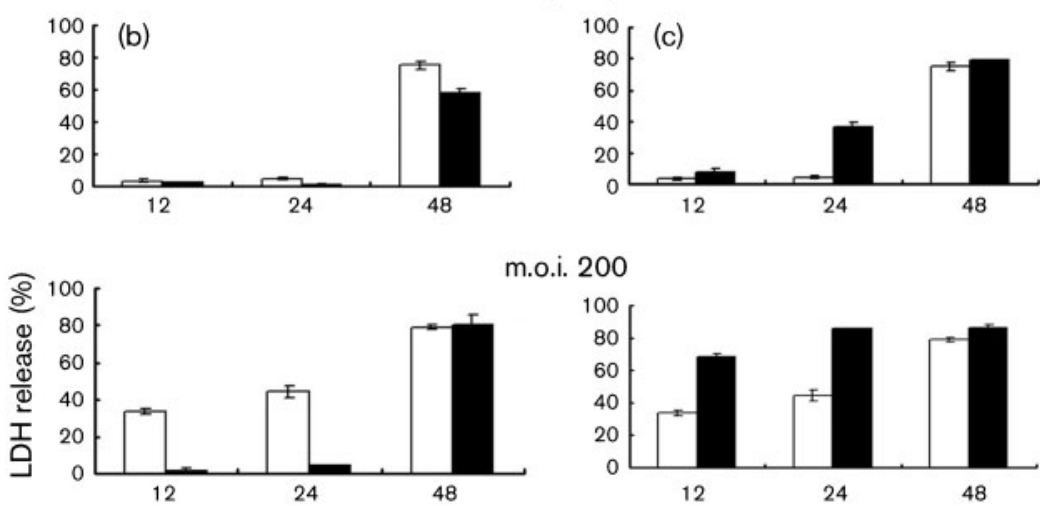

m.o.i. 200

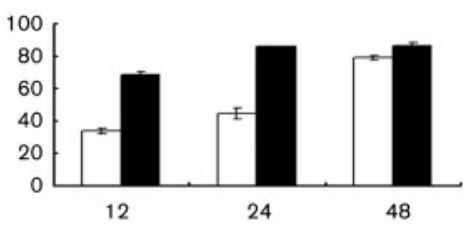

m.o.i. 2000
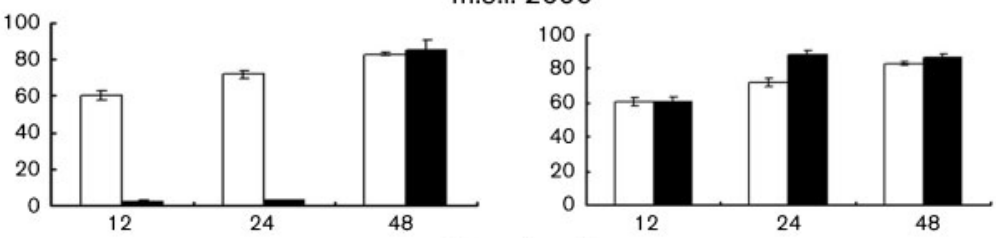

Time (h p.i.)
Fig. 2. T4SS-dependent cytotoxicity of smooth strain for macrophage. (a) J774A.1 cells were infected with strain BM at m.o.i.s of 20 (white bars), 200 (black bars) and 2000 (grey bars). (b) J774A.1 cells were infected with strains BM (white bars) and $B M \Delta$ virB (black bars) at m.o.i.s of 20, 200 and 2000. (c) J774A. 1 cells were infected with strains BM (white bars) and BM-VIR (black bars) at m.o.i.s of 20, 200 and 2000. The supernatant was collected at the indicated time points, and the $\mathrm{LDH}$ released by the infected cells was detected by using the CytoTox 96 nonradioactive cytotoxicity assay. The data shown are the means $\pm S D$ of a representative experiment that was repeated twice. were dead because of the long period of incubation. These results implied that the smooth strain is cytotoxic for macrophages at higher levels of infection.

\section{Cytotoxicity of the smooth strain for macrophages is virB-dependent}

Previous studies indicated that rough mutants are cytotoxic for macrophages, and this is presumed to be the result of exposure of the T4SS on the surface. Subsequent experiments have shown that cytotoxicity is T4SS-dependent. To test whether the cytotoxicity of the smooth strain for macrophages is also related to $\operatorname{virB}$ expression, macrophages were infected with strains BM and $B M \Delta v i r B$, and the release of $\mathrm{LDH}$ was quantified. When infected at an m.o.i. of 200 , at 12 h p.i. $33.53 \%$ of $\mathrm{LDH}$ was released in BM-infected macrophages; however, for cells infected with $\mathrm{BM} \Delta \mathrm{virB}$, only $1.47 \% \mathrm{LDH}$ was released, a reduction of about 23 -fold. Even when infected with $\mathrm{BM} \Delta \mathrm{virB}$ at an m.o.i. of 2000 , the macrophages released only very low levels of LDH. The high level of LDH released in macrophages infected with both $\mathrm{BM}$ and $\mathrm{BM} \Delta$ virB at $48 \mathrm{~h}$ p.i. further indicated that $\mathrm{LDH}$ release after this time is an effect of the long incubation period. As shown in Fig. 2(b), all macrophages infected with $B M \Delta$ virB released very low levels of $\mathrm{LDH}$ and, furthermore, there was no m.o.i.-dependent increase in LDH $(P>0.05)$, in contrast to strain BM. These results indicated that the cytotoxicity of the smooth strain for macrophages is dependent on expression of virB.

\section{Overexpression of virB in Brucella is cytopathic for macrophages at low m.o.i.}

The experiments above indicate that when infected at higher levels of m.o.i., macrophages release higher levels of $\mathrm{LDH}$, and when $\operatorname{vir} B$ is inactivated, the release of $\mathrm{LDH}$ is greatly reduced. We presumed that at high m.o.i., plenty of Brucella cells reside in the macrophages and, therefore, large quantities of effector proteins are secreted into the host cells through the T4SS, disrupting signal networks and leading to the cytotoxicity. This is supported by the 
observation that the virB mutant did not induce a high level of LDH release, even at high m.o.i. To further test whether LDH release is related to overexpression of $\operatorname{virB}$, the $\operatorname{vir} B$ overexpression strain BM-VIR was used to infect macrophages. As shown in Fig. 2(c), even at the lowest m.o.i. of 20 , macrophages infected with strain BM-VIR released 7.8 and $36.9 \% \mathrm{LDH}$ at 12 and $24 \mathrm{~h}$ p.i., respectively, three and nine times higher than the levels released in macrophages infected with strain BM (3.2 and $4.2 \%$ at 12 and $24 \mathrm{~h}$ p.i., respectively) at the two time points $(P<0.01)$, indicating that $\mathrm{LDH}$ release is significantly increased in macrophages infected with strain BMVIR. When infected at an m.o.i. of 200, the level of LDH release in macrophages infected with strain BM-VIR at $12 \mathrm{~h}$ p.i. was $68.6 \%$, twice that of macrophages infected with strain $\mathrm{BM}(33.5 \%)(P<0.01)$. At $24 \mathrm{~h}$ p.i., $\mathrm{LDH}$ release for BM-infected macrophages was $44.3 \%$, but for cells infected with strain BM-VIR it reached the plateau level of over $85 \%$, implying that overexpression of virB shortened the time required to reach the $\mathrm{LDH}$ release plateau. However, when macrophages were infected with strain BM-VIR at an m.o.i. of 2000, the level of $\mathrm{LDH}$ release $(60.6 \%)$ was comparable to that of cells infected with strain $\mathrm{BM}(60.2 \%)$ at $12 \mathrm{~h}$ p.i. $(P>0.05)$. These data indicate that the cytotoxicity of smooth strains is induced by overexpression of virB. Based on these results, it can be reasoned that cytotoxicity of wild-type strains for macrophages at high levels of m.o.i. might be mediated by overexpression of $v i r B$, for the number of bacterial cells in a single macrophage cell is increased, and higher levels of effector proteins are secreted in this cell.

\section{$\mathrm{C}_{12}$-HSL inhibits Brucella cytotoxicity for macrophages in a virB-dependent manner}

Quorum sensing is a cell-density-dependent gene expression regulation mechanism, mediated by acyl homoserine lactone (AHL) or auto-inducer 2 (AI-2) signal molecules. The AHL-type molecule $\mathrm{C}_{12}$-HSL has been identified in supernatants of Brucella cultures (Delrue et al., 2005; Taminiau et al., 2002). This molecule has been shown to inhibit expression of the virB operon. Subsequent experiments indicated that the quorum sensing regulator $v j b R$ regulates expression of virB (Taminiau et al., 2002). To determine whether $\mathrm{C}_{12}$-HSL downregulates expression of $\operatorname{vir} B$ and accordingly inhibits virB-dependent cytotoxicity of Brucella, macrophages were infected with strains BM, $\mathrm{BM} \Delta$ virB or BM-VIR at an m.o.i. of 200 and then treated with different concentrations of $\mathrm{C}_{12}$-HSL. Since the survival and replication of Brucella are not affected by $\mathrm{C}_{12}$-HSL treatment, even at the highest concentrations, $\mathrm{C}_{12}$-HSL does not appear to be bactericidal or bacteriostatic to Brucella, and therefore cytotoxicity effects could be considered to be mediated by $\mathrm{C}_{12}$-HSL. As shown in Fig. 3, when $\mathrm{C}_{12}$-HSL was added into the media of cells infected with strain BM, the cytotoxicity for macrophages was inhibited in a dose-dependent manner: the higher the concentration of $\mathrm{C}_{12}-\mathrm{HSL}$, the greater the inhibition of

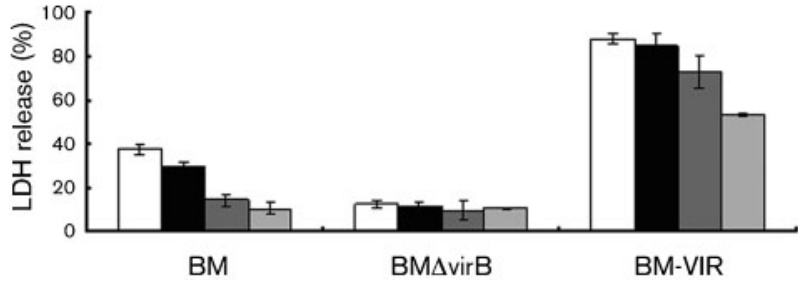

Fig. 3. Inhibition of cytotoxicity by $\mathrm{C}_{12}-\mathrm{HSL}$ in a virB-dependent manner. J774.A1 macrophages were infected with strains $B M$, $\mathrm{BM} \Delta$ virB and BM-VIR at an m.o.i. of 200. $\mathrm{C}_{12}-\mathrm{HSL}$ was added to the culture media at various concentrations $\left(\mu \mathrm{g} \mathrm{ml}^{-1}\right.$ : white bars, 0 ; black bars, 0.126; dark grey bars, 0.63; light grey bars, 1.26) and the release of $\mathrm{LDH}$ was measured at $24 \mathrm{~h}$ p.i. The data shown are the means \pm SD of a representative experiment that was repeated twice.

cytotoxicity $(P<0.01)$. When the concentration of $\mathrm{C}_{12}$-HSL was increased to $1.26 \mu \mathrm{g} \mathrm{ml}^{-1}$, the level of cytotoxicity was comparable to that of $B M \Delta v i r B$-infected macrophages, indicating that cytotoxicity was completely inhibited. For the virB mutant $\mathrm{BM} \Delta \mathrm{virB}$, no or only trace cytotoxicity was observed, and therefore no inhibition was observed. For strain BM-VIR, cytotoxicity was also inhibited by $\mathrm{C}_{12}-\mathrm{HSL}$ in a dose-dependent manner $(P<0.01)$. However, even when treated with the highest concentration of $\mathrm{C}_{12}-\mathrm{HSL}$, the level of $\mathrm{LDH}$ release $(53.3 \%)$ was higher than that of untreated strain BM (37.4\%), indicating that cytotoxicity could not be completely inhibited in BM-VIR. These results indicated that $\mathrm{C}_{12}$-HSL inhibits Brucella cytotoxicity for macrophages in a virB-dependent manner. For the virB overexpression strain, the cytotoxicity could not be completely inhibited, probably because of the increased level of transcription of $\operatorname{vir} B$.

\section{B. melitensis virB overexpression strain exhibits reduced survival in macrophages and mice}

Intracellular bacteria have developed various strategies to evade elimination by host cells. On one hand, intracellular bacteria attempt to adapt to the hostile environments in the host cell. On the other hand, a number of bacteria, including Brucella, are found to inhibit host-cell apoptosis and death, which presumably promotes bacterial survival and replication in host cells (Gross et al., 2000). T4SS is an essential determinant for Brucella intracellular survival. The results described above indicate that overexpression of virB in Brucella is cytotoxic for macrophages, and we therefore speculated that such overexpression of virB might be detrimental for Brucella intracellular survival.

To evaluate the effects of overexpression of virB on Brucella intracellular survival, J774.A1 cells were infected with strains BM-VIR, BM $\Delta$ virB and BM. When macrophages were infected with strain BM, about $90 \%$ of the invading bacteria were killed by the macrophages within the first $4 \mathrm{~h}$ (Fig. 4a). Thereafter, an increase in intracellular bacteria 
(a)

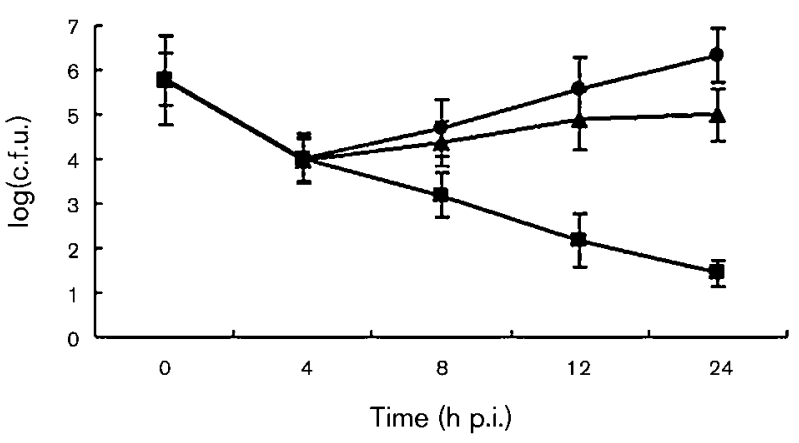

(b)

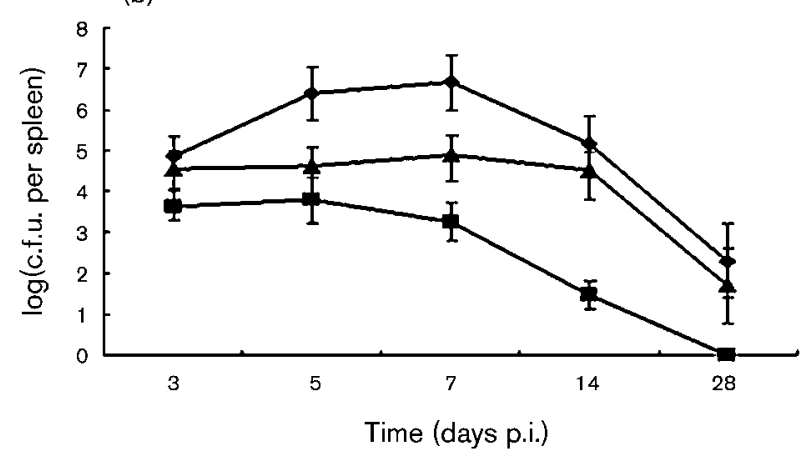

(c)

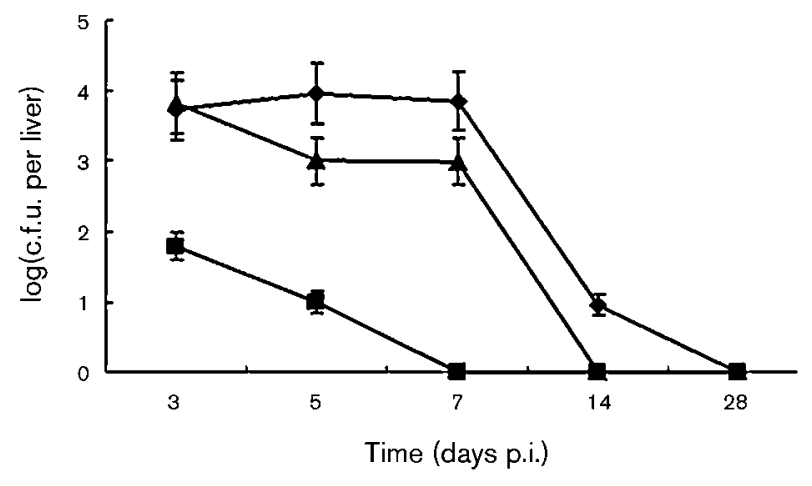

Fig. 4. Survival of virB overexpression strain in macrophages (a) and mice (b, c). (a) J774.A1 cells were infected with strains BM $(\diamond), B M \Delta v i r B(\boldsymbol{\square})$ and BM-VIR $(\boldsymbol{\Lambda})$ at an m.o.i. of $200: 1$. At the indicated time points p.i., cells were lysed and bacteria were enumerated by plating serial dilutions on TSA plates. $(b, c)$ Five BALB/c mice were injected intraperitoneally with $5 \times 10^{7}$ c.f.u. BM $(\diamond), B M \Delta v i r B(\boldsymbol{\square})$ and BM-VIR $(\boldsymbol{\Lambda})$ per group. Infected mice were killed by $\mathrm{CO}_{2}$ asphyxiation at $3,5,7,14$ and 28 days p.i. Spleens and livers were aseptically removed and homogenized with $1 \mathrm{ml}$ PBS. Bacteria cell number was counted by plating serial dilutions on TSA plates.

was observed, resulting from replication of the surviving bacteria. For strains BM-VIR and BM $\Delta$ virB, there was an identical decrease in live bacteria in the first $4 \mathrm{~h}$ p.i. However, after this time point, the two strains seemed to have a different fate from strain BM. The number of intracellular live bacteria of strain $\mathrm{BM} \Delta \mathrm{virB}$ progressively decreased $(P<0.01)$, indicating a loss of survival capability in macrophages. For strain BM-VIR, the number of intracellular live bacteria did not increase sharply as it did with strain BM, indicating that although strain BMVIR could survive in macrophages, there was a decreased level of intracellular survival. To further analyse the virulence of the virB overexpression strain, we compared the survival of strains $B M, B M \Delta v i r B$ and $B M-V I R$ in $\mathrm{BALB} / \mathrm{c}$ mice. Strain BM-VIR showed similar kinetics of infection in the spleen and liver to strain BM, with maximum colonization at 5 days p.i., decreasing slowly thereafter until the end of the experiment at 28 days p.i. However, the maximum level of colonization of strain BMVIR was much lower than that of strain BM (Fig. 4b, c) $(P<0.01)$. Therefore, although strain BM-VIR can survive in macrophages and mouse spleen and liver cells, it exhibits a decreased capability to survive.

\section{Overexpression of virB does not affect survival of Brucella under stress conditions}

Overexpression of virB in Brucella is cytopathic for macrophages and decreases the survival capability of Brucella in macrophages and mouse spleen and liver cells. The reason for this is presumably that overexpression of virB leads to increased secretion of effector proteins and excessive interference with the signal network of host cell. To survive in host cells, in addition to interfering with host cell signals, bacteria need to adapt to the intracellular environment. To test whether overexpression of $\operatorname{vir} B$ affects Brucella adaptation to the intracellular environment, the survival of strains BM, BM $\Delta$ virB, and BM-VIR under various stress conditions simulating intracellular environments was analysed and compared. The virB mutant $\mathrm{BM} \Delta$ virB showed reduced survival under the stress conditions tested compared with strain BM $(P<0.01)$, implying a role for virB in the ability of Brucella to resist these stresses. However, compared to strain BM, the survival of strain BM-VIR under these conditions was not very different (Fig. 5) $(P>0.05)$. This indicates that expression of virB is important for the ability of Brucella to adapt to stress conditions and thus probably the intracellular environment, but overexpression of virB has little effect on this ability. These data indirectly imply that the cytotoxicity of the virB-overexpressing strain is mediated by an increased level of effector proteins.

\section{virB in strain BM-VIR is highly transcribed during macrophage infection and under stress conditions}

The above experiments showed that the virB-overexpression strain is more cytotoxic for macrophages, and this effect is most probably mediated by increased expression of virB. To test whether the virB is overexpressed in strain BM-VIR during host-cell infection and under stress conditions, the relative levels of transcription of $\operatorname{vir} B$ were analysed by qRT-PCR. During macrophage infection, the 


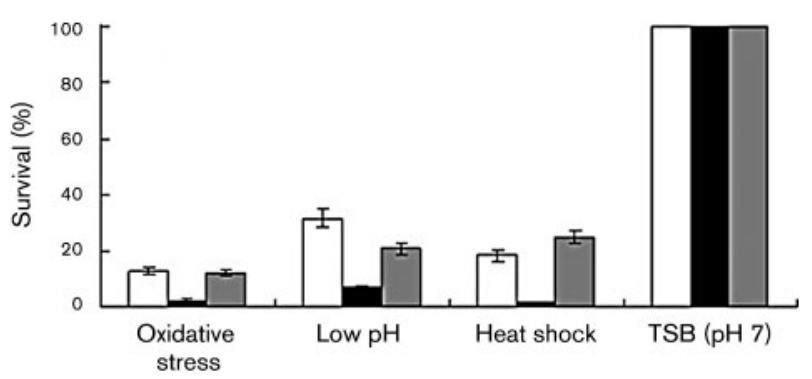

Fig. 5. Overexpression of virB had little effect on Brucella survival under stress conditions. Strains BM (white bars), BM $\Delta$ virB (black bars) and BM-VIR (grey bars) were first cultured in TSB to exponential phase and then subjected to the appropriate stress conditions as described in the text. After treatment, the surviving bacteria were enumerated by plating serial dilutions onto TSA plates. The data shown are the means $\pm S D$ of a representative experiment that was repeated twice.

transcription profile of virB in strain BM-VIR was identical to that of strain $\mathrm{BM}$, being activated immediately postinfection, peaking at $12 \mathrm{~h}$ p.i. and then downregulated. However, the level of transcription of virB in strain BMVIR was much higher than that in strain $\mathrm{BM}(P<0.01)$, being about fourfold higher at $0 \mathrm{~h}$ p.i. and $12 \mathrm{~h}$ p.i., and 10 -fold higher by $24 \mathrm{~h}$ p.i. (Fig. 6). This elevated level of transcription is related to the increased cytotoxicity for macrophages, but inversely related to the decreased survival of strain BM-VIR in macrophages. In the in vitro stress study, virB in strain BM-VIR was transcribed at a higher level than in strain BM under all stress conditions tested $(P<0.01)$, indicating that $\operatorname{vir} B$ was overexpressed under these conditions, but this has no bearing on its ability to survive under these conditions.

\section{virB is transcribed in a cell-density-dependent manner both in vitro and during macrophage infection}

It is known that the number of Brucella cells in macrophages is maintained at a relatively low level. The experiments described above showed that with a higher m.o.i., higher cytotoxicity was observed. This implies the possibility of cell-density-dependent expression regulation of $v i r B$. Further evidence to support this possibility comes from the fact that $\operatorname{vir} B$ is regulated by $v j b R$, a quorum sensing system which senses and responds to cell density. Therefore, we analysed the transcription of $\operatorname{vir} B$ at different growth stages. Growth curves showed that strain BM-VIR had a similar growth curve to strain BM, but the growth rate was lower (Fig. 7a). However, transcription of virB in the two strains seemed to be very different. In strain BM, $\operatorname{vir} B$ was transcribed at the highest level at low cell densities (early exponential phase), and as cell density increased, transcription decreased. However, for strain BM-VIR, transcription of virB increased as cell density increased

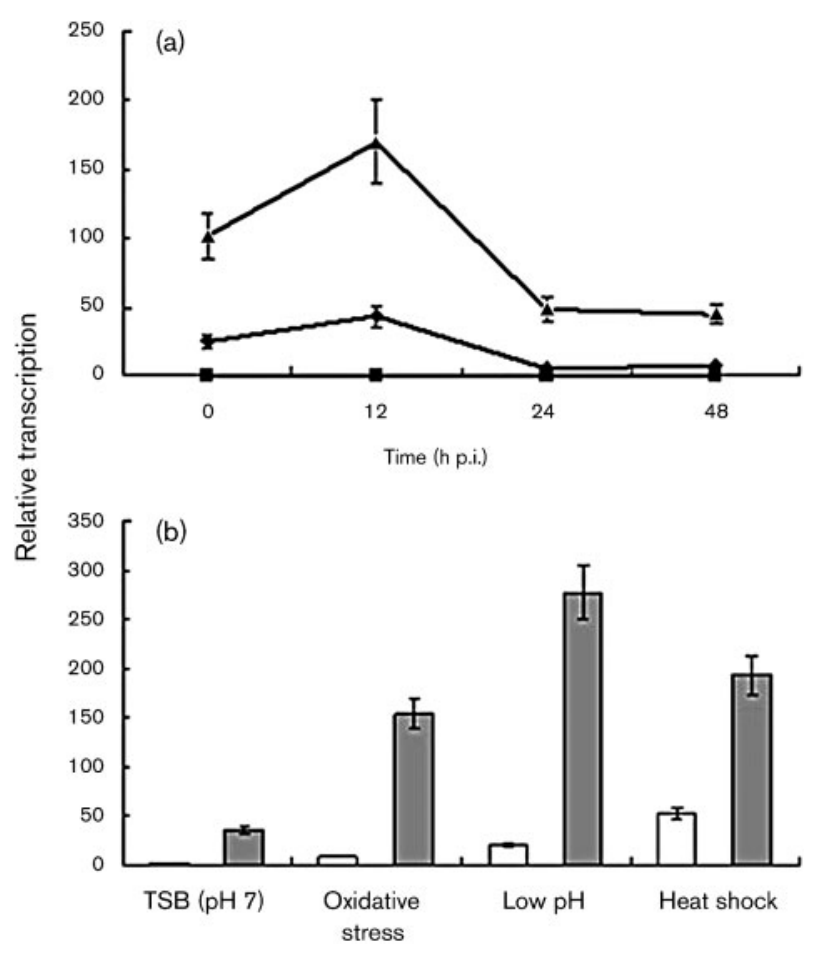

Fig. 6. Overexpression of virB in strain BM-VIR during macrophage infection (a) and under stress conditions (b). (a) Macrophage cells were infected with strains BM $(\bullet), B M \Delta$ virB (ם) and BM-VIR (A). At different times (0, 12, 24 and $48 \mathrm{~h}$ p.i.), RNA was isolated from the infection mixtures and reverse transcribed into cDNA. Transcription of virB genes was then quantified by qRT-PCR. (b) Strains BM (white bars), BM $\Delta$ virB (bars not visible) and BM-VIR (grey bars) were cultured in TSB to early exponential phase $\left(\mathrm{OD}_{600}=0.6\right)$ at $37^{\circ} \mathrm{C}$ and then subjected to different stress conditions. RNA was isolated and the transcription of virB was quantified by qRT-PCR.

from early to late exponential phase, and at stationary phase the transcription of $\operatorname{vir} B$ was downregulated. At high cell density, transcription of virB in strain BM-VIR was much higher than that observed in strain BM $(P<0.01)$. Transcription of virB during macrophage infection peaked at $12 \mathrm{~h}$ p.i., and then decreased at $24-48 \mathrm{~h}$ p.i. Combined with the change in the number of intracellular bacteria over the infection period, it can be inferred that the transcription of virB during macrophage infection is cell-densitydependent.

\section{DISCUSSION}

The T4SS has been identified in many pathogenic bacteria, such as Legionella pneumophila, A. tumefaciens, Helicobacter pylori and Bartonella spp. These bacteria use the T4SS apparatus to interfere with host-cell function by secretion of effector proteins (Cascales \& Christie, 2003). As an intracellular bacterial pathogen, Brucella needs to adapt to the intracellular environment and interfere with 

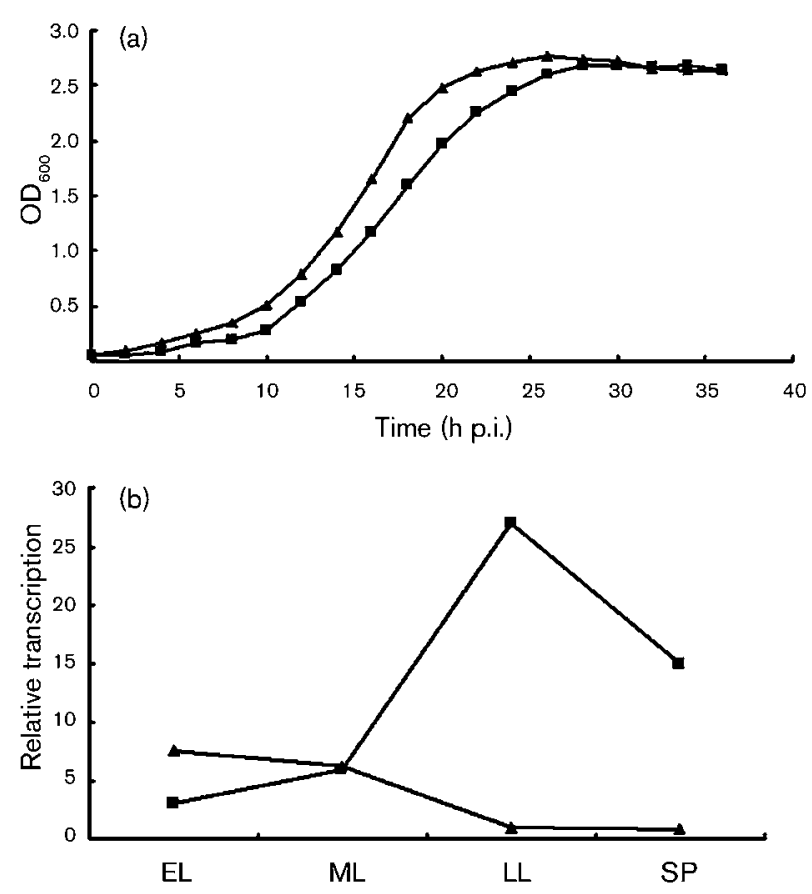

Fig. 7. Growth-dependent transcription of virB. (a) Growth curve of strains BM ( $\boldsymbol{\Delta})$ and BM-VIR $(\boldsymbol{\square})$ in TSB. OD 600 was monitored at $2 \mathrm{~h}$ intervals. (b) Transcription of virB8 during different growth phases. Strains BM $(\boldsymbol{A})$ and BM-VIR $(\boldsymbol{\square})$ were cultured in TSB, and then samples of each were grown to early exponential (EL), mid-exponential $(\mathrm{ML})$, late exponential (LL) and stationary phases (SP).

host-cell function for survival and replication. The T4SS of Brucella has been shown to be an essential virulence determinant, which plays important roles in phagosomelysosome fusion inhibition and replication vacuole formation (Celli et al., 2003; O'Callaghan et al., 1999). The T4SS seems to play different roles in different Brucella species. For smooth strains, T4SS is essential for intracellular survival and plays a very important role in the inhibition of host-cell apoptosis. However, for some rough strains, the T4SS induces cytotoxicity for macrophages, implying complex regulation of the T4SS in different Brucella backgrounds (Freeman \& Rumack, 1964; Pei et al., 2008).

Cytotoxicity in macrophages induced by rough Brucella mutants requires bacterial protein synthesis, and the T4SS is critical for this cytotoxicity. One explanation of the cytotoxicity of the rough mutant is assumed to be the increased secretion of toxin (Pei et al., 2008). In the present study, we found for the first time that smooth strains also induce cytotoxicity in macrophages. The cytotoxicity of smooth strains for macrophages occurs at high levels of m.o.i. Inactivation of the T4SS eliminated cytotoxicity; in contrast, overexpression of the T4SS increased cytotoxicity even at low levels of m.o.i. These data indicate that the cytotoxicity of smooth strains for macrophages is also T4SS-dependent.
To determine whether smooth strains are cytotoxic for macrophages, J774A.1 cells were infected with smooth strain BM at different m.o.i.s. To our surprise, when macrophages were infected with a high m.o.i., cytotoxicity was observed. Furthermore, the cytotoxicity was positively correlated with m.o.i., implying that the increased numbers of bacteria in the host cells might be harmful to the host. The length of time post-infection seemed to affect host cells detrimentally; when the infection was prolonged to $48 \mathrm{~h}$, all the macrophages infected at different m.o.i.s showed high levels of cytotoxicity (Fig. 2a). This provided further evidence that large numbers of bacteria are harmful for the host cell. Previous studies have also shown that optimal numbers of bacteria are required for Brucella to protect infected and neighbouring host cells, implying that bacteria cell numbers in the host are probably tightly regulated (Delrue et al., 2005; Pei et al., 2008).

Following the observation that smooth strains are cytotoxic for macrophages, we then tested whether this effect is related to the T4SS, as seen in the rough strains. As shown in Fig. 2(b), the virB mutant did not induce high levels of cytotoxicity in macrophages at any m.o.i. by 12 and $24 \mathrm{~h}$ p.i.; furthermore, there were no differences in the level cytotoxicity for the three m.o.i.s. The cytotoxicity of strain $\mathrm{BM} \Delta$ virB for macrophages at $48 \mathrm{~h}$ p.i. may result from a nutritionally deprived environment due to the long incubation time, as found with strain BM at a low m.o.i. of 20. Therefore, based on these results, it can be concluded that the cytotoxicity of the smooth strain is virBdependent. Pei et al. (2006) found that the cytotoxicity of rough mutants of Brucella ovis for macrophages requires protein synthesis. Their subsequent study showed that this cytotoxicity is T4SS-dependent (Pei et al., 2008). In our present study, we found that the smooth strain of $B$. melitensis is also cytotoxic for macrophages. This implies that cytotoxicity for macrophages is not unique to rough mutants, and that virB-dependent cytotoxicity might be a common phenomenon among different Brucella strains.

The cytotoxicity of the rough mutant for macrophages is predicted to result from the increased secretion of toxin into the host cell by the bacteria because of increased exposure of the T4SS in the rough mutant. Based on the mechanism of the T4SS, these toxins are probably effector proteins. Thus we speculated that the cytotoxicity of the smooth strain might also result from overexpression of virB. As our results show, when infected with virBoverexpressing strain BM-VIR, even at a low m.o.i. of 20 (at which little cytotoxicity of strain BM was observed), the infected macrophage exhibited a high degree cytotoxicity. The time to reach the $\mathrm{LDH}$ release plateau was also shortened with strain BM-VIR. This indicated that overexpression of $v i r B$ increases cytotoxicity of Brucella for macrophages. Both overexpression of virB in strain BMVIR and a high m.o.i. of strain BM induced a high degree of cytotoxicity, the common characteristic of which is that virB is expressed at elevated levels. Thus the main cause of cytotoxicity of Brucella for macrophages is elevated 
expression of virB, and most probably the increased secretion of effector proteins. This is confirmed by the result that $\mathrm{C}_{12}$-HSL inhibits expression of virB, and also inhibits cytotoxicity of Brucella for macrophages (Fig. 3).

In $B$. suis, virB is regulated in a growth-phase-dependent manner, with $\operatorname{vir} B$ transcription being reduced in late exponential phase. Delrue et al. (2005) observed that the level of virB mRNA in B. melitensis decreased significantly upon reaching stationary phase. In the present study, we found that transcription of virB is also growth-dependent in B. melitensis strain BM. Transcription of virB peaks at early exponential growth phase, and then as the cell density increases, transcription decreases (Fig. $7 \mathrm{~b}$ ). Addition of $\mathrm{C}_{12}$-HSL inhibits virB transcription in B. melitensis and this is independent of growth stage. Inactivation of $v j b R$ downregulates transcription of virB. The inhibition effect of $\mathrm{C}_{12}$-HSL on transcription of $\operatorname{virB}$ is mediated by $v j b R$, indicating that $v j b R$ regulates the expression of $v i r B$. The regulation of $v i r B$ by $v j b R$ appears to be correlated with intracellular survival, for increased bacterial numbers are detrimental for host cells. Interestingly, we found that $\operatorname{vir} B$ also affects transcription of $v j b R$ in a growth-phasedependent manner. Therefore, $v j b R$ and $v i r B$ regulate each other, forming a positive regulation circuit (Wang et al., 2009). Both vjbR and virB mutants could not replicate in host cells, nor cause chronic infection in mice. Thus the numbers of intracellular Brucella cells are tightly controlled and closely related to intracellular survival.

Inactivation of the T4SS in the smooth strain resulted in rapid clearance of the mutant by host cells. However, the elimination of T4SS expression in rough mutants resulted in enhanced replication and persistence, suggesting that the T4SS is not required for rough mutant intracellular trafficking and survival in macrophages. Therefore, it is possible that rough Brucella strains invade macrophages by a different pathway from smooth strains, which influences both the intracellular trafficking and the ultimate fate of rough organisms (Gorvel \& Moreno, 2002; Jimenez de Bagues et al., 2004; Pei et al., 2006). A recent study showed that two proteins, VceA and $\mathrm{VceC}$, were translocated into host cells by the Brucella T4SS (de Jong et al., 2008). Translocation of VceC by B. abortus into J774A.1 cells resulted in lysis of the host cells, indicating that this protein mediates the cytotoxicity effect for macrophages. At an m.o.i. of 500 , at $11 \mathrm{~h}$ p.i., only $30 \%$ cytotoxicity for macrophages was observed. This indicated that there are other effector proteins that are involved in the cytotoxicity (de Jong et al., 2008). In other bacteria, a large number of effector proteins involved in different functional categories are secreted via the T4SS. Therefore, VceC and VceA will not be the only effector proteins secreted by the T4SS of Brucella. It will be very interesting to construct a VceC deletion mutant and analyse the cytotoxicity of this mutant for macrophages.

The virB mutant could not replicate in macrophages and the $\operatorname{vir} B$ overexpression strain was cytotoxic for macro- phage, indicating that the expression of virB is tightly regulated. The virB mutant showed increased sensitivity to in vitro stress conditions, implying that virB plays important roles in the adaptation of Brucella to intracellular environments. With comparative proteomic methods, we found that inactivation of $v i r B$ resulted in great changes in gene expression, including many involved in bacterial intracellular survival. The major membrane proteins, such as Omp25 and Omp31, were also downregulated in the virB mutant (Wang et al., 2009). The downregulation of outer-membrane proteins may explain the increased sensitivity of the mutant to stress. We speculate that some of these differentially expressed proteins might be the effector proteins of the T4SS. At present, we are trying to identify these effector proteins.

In summary, this report provides evidence that smooth strains of Brucella are toxic for macrophages at a high infection m.o.i., and this cytotoxicity is also T4SSdependent. Inactivation of the T4SS eliminated intracellular survival capability, and overexpression of the T4SS increased cytotoxicity but not sensitivity to intracellular stress conditions, indicating that cytotoxicity is mediated by increased secretion of effector proteins and that virB is tightly regulated during survival in macrophages. Transcription of virB both under stress conditions and during macrophage infection showed that $\operatorname{vir} B$ is tightly regulated in a cell-density-dependent manner. Therefore, it is possible that the secretion of effector proteins is also regulated in this way. The identification of more effector proteins of the T4SS and research into their functions will provide more detailed answers.

\section{ACKNOWLEDGEMENTS}

This work was supported by the National Natural Science Foundation of China (Grant No. 30600024, to Z. L. C.) and the National High Technology Research and Development Program of China (Grant No. 2007AA02Z412, to Z. L.C.).

\section{REFERENCES}

Alvarez-Martinez, M. T., Machold, J., Weise, C., Schmidt-Eisenlohr, H., Baron, C. \& Rouot, B. (2001). The Brucella suis homologue of the Agrobacterium tumefaciens chromosomal virulence operon chvE is essential for sugar utilization but not for survival in macrophages. J Bacteriol 183, 5343-5351.

Boschiroli, M. L., Ouahrani-Bettache, S., Foulongne, V., MichauxCharachon, S., Bourg, G., Allardet-Servent, A., Cazevieille, C., Lavigne, J. P., Liautard, J. P. \& other authors (2002a). Type IV secretion and Brucella virulence. Vet Microbiol 90, 341-348.

Boschiroli, M. L., Ouahrani-Bettache, S., Foulongne, V., MichauxCharachon, S., Bourg, G., Allardet-Servent, A., Cazevieille, C., Liautard, J. P., Ramuz, M. \& O'Callaghan, D. (2002b). The Brucella suis virB operon is induced intracellularly in macrophages. Proc Natl Acad Sci U S A 99, 1544-1549.

Cascales, E. \& Christie, P. J. (2003). The versatile bacterial type IV secretion systems. Nat Rev Microbiol 1, 137-149. 
Celli, J., de Chastellier, C., Franchini, D. M., Pizarro-Cerda, J., Moreno, E. \& Gorvel, J. P. (2003). Brucella evades macrophage killing via VirB-dependent sustained interactions with the endoplasmic reticulum. J Exp Med 198, 545-556.

Celli, J., Salcedo, S. P. \& Gorvel, J. P. (2005). Brucella coopts the small GTPase Sar1 for intracellular replication. Proc Natl Acad Sci U S A 102, 1673-1678.

Comerci, D. J., Martinez-Lorenzo, M. J., Sieira, R., Gorvel, J. P. \& Ugalde, R. A. (2001). Essential role of the VirB machinery in the maturation of the Brucella abortus-containing vacuole. Cell Microbiol 3, 159-168.

de Jong, M. F., Sun, Y. H., den Hartigh, A. B., van Dijl, J. M. \& Tsolis, R. M. (2008). Identification of VceA and VceC, two members of the VjbR regulon that are translocated into macrophages by the Brucella type IV secretion system. Mol Microbiol 70, 1378-1396.

Delrue, R. M., Deschamps, C., Leonard, S., Nijskens, C., Danese, I., Schaus, J. M., Bonnot, S., Ferooz, J., Tibor, A. \& other authors (2005). A quorum-sensing regulator controls expression of both the type IV secretion system and the flagellar apparatus of Brucella melitensis. Cell Microbiol 7, 1151-1161.

Foulongne, V., Bourg, G., Cazevieille, C., Michaux-Charachon, S. \& O'Callaghan, D. (2000). Identification of Brucella suis genes affecting intracellular survival in an in vitro human macrophage infection model by signature-tagged transposon mutagenesis. Infect Immun 68, 1297-1303.

Freeman, B. A. \& Rumack, B. H. (1964). Cytopathogenic effect of Brucella spheroplasts on monocytes in tissue culture. J Bacteriol 88, 1310-1315.

Gorvel, J. P. \& Moreno, E. (2002). Brucella intracellular life: from invasion to intracellular replication. Vet Microbiol 90, 281-297.

Gross, A., Terraza, A., Ouahrani-Bettache, S., Liautard, J. P. \& Dornand, J. (2000). In vitro Brucella suis infection prevents the programmed cell death of human monocytic cells. Infect Immun 68, 342-351.

Hong, P. C., Tsolis, R. M. \& Ficht, T. A. (2000). Identification of genes required for chronic persistence of Brucella abortus in mice. Infect Immun 68, 4102-4107.

Jimenez de Bagues, M. P., Terraza, A., Gross, A. \& Dornand, J. (2004). Different responses of macrophages to smooth and rough Brucella spp.: relationship to virulence. Infect Immun 72, 2429-2433.

Kim, S., Watarai, M., Makino, S. \& Shirahata, T. (2002). Membrane sorting during swimming internalization of Brucella is required for phagosome trafficking decisions. Microb Pathog 33, 225-237.

Kovach, M. E., Elzer, P. H., Hill, D. S., Robertson, G. T., Farris, M. A., Roop, R. M., II \& Peterson, K. M. (1995). Four new derivatives of the broad-host-range cloning vector pBBR1MCS, carrying different antibiotic-resistance cassettes. Gene 166, 175-176.

Lestrate, P., Delrue, R. M., Danese, I., Didembourg, C., Taminiau, B., Mertens, P., De Bolle, X., Tibor, A., Tang, C. M. \& Letesson, J. J. (2000). Identification and characterization of in vivo attenuated mutants of Brucella melitensis. Mol Microbiol 38, 543-551.

Livak, K. J. \& Schmittgen, T. D. (2001). Analysis of relative gene expression data using real-time quantitative PCR and the $2^{-\Delta \Delta C_{\mathrm{T}}}$ method. Methods 25, 402-408.
Mota, L. J., Journet, L., Sorg, I., Agrain, C. \& Cornelis, G. R. (2005). Bacterial injectisomes: needle length does matter. Science 307, 1278.

Nijskens, C., Copin, R., De Bolle, X. \& Letesson, J. J. (2008). Intracellular rescuing of a $B$. melitensis $16 \mathrm{M}$ virB mutant by coinfection with a wild type strain. Microb Pathog 45, 134-141.

O'Callaghan, D., Cazevieille, C., Allardet-Servent, A., Boschiroli, M. L., Bourg, G., Foulongne, V., Frutos, P., Kulakov, Y. \& Ramuz, M. (1999). A homologue of the Agrobacterium tumefaciens VirB and Bordetella pertussis Ptl type IV secretion systems is essential for intracellular survival of Brucella suis. Mol Microbiol 33, 1210-1220.

Pei, J. \& Ficht, T. A. (2004). Brucella abortus rough mutants are cytopathic for macrophages in culture. Infect Immun 72, 440-450.

Pei, J., Turse, J. E., Wu, Q. \& Ficht, T. A. (2006). Brucella abortus rough mutants induce macrophage oncosis that requires bacterial protein synthesis and direct interaction with the macrophage. Infect Immun 74, 2667-2675.

Pei, J., Wu, Q., Kahl-McDonagh, M. \& Ficht, T. A. (2008). Cytotoxicity in macrophages infected with rough Brucella mutants is type IV secretion system dependent. Infect Immun 76, 30-37.

Pizarro-Cerda, J., Meresse, S., Parton, R. G., van der Goot, G., SolaLanda, A., Lopez-Goni, I., Moreno, E. \& Gorvel, J. P. (1998). Brucella abortus transits through the autophagic pathway and replicates in the endoplasmic reticulum of nonprofessional phagocytes. Infect Immun 66, 5711-5724.

Sieira, R., Comerci, D. J., Sanchez, D. O. \& Ugalde, R. A. (2000). A homologue of an operon required for DNA transfer in Agrobacterium is required in Brucella abortus for virulence and intracellular multiplication. J Bacteriol 182, 4849-4855.

Starr, T., Ng, T. W., Wehrly, T. D., Knodler, L. A. \& Celli, J. (2008). Brucella intracellular replication requires trafficking through the late endosomal/lysosomal compartment. Traffic 9, 678-694.

Taminiau, B., Daykin, M., Swift, S., Boschiroli, M. L., Tibor, A., Lestrate, P., De Bolle, X., O'Callaghan, D., Williams, P. \& Letesson, J. J. (2002). Identification of a quorum-sensing signal molecule in the facultative intracellular pathogen Brucella melitensis. Infect Immun 70, 3004-3011.

Teixeira-Gomes, A. P., Cloeckaert, A. \& Zygmunt, M. S. (2000). Characterization of heat, oxidative, and acid stress responses in Brucella melitensis. Infect Immun 68, 2954-2961.

Wang, Y., Chen, Z., Qiao, F., Ying, T., Yuan, J., Zhong, Z., Zhou, L., Du, X., Wang, Z. \& other authors (2009). Comparative proteomics analyses reveal the $\operatorname{vir} B$ of $B$. melitensis affects expression of intracellular survival related proteins. PLoS One 4, e5368.

Watarai, M., Makino, S., Fujii, Y., Okamoto, K. \& Shirahata, T. (2002). Modulation of Brucella-induced macropinocytosis by lipid rafts mediates intracellular replication. Cell Microbiol 4, 341-355.

West, N. P., Sansonetti, P., Mounier, J., Exley, R. M., Parsot, C., Guadagnini, S., Prévost, M. C., Prochnicka-Chalufour, A., Delepierre, M. \& other authors (2005). Optimization of virulence functions through glucosylation of Shigella LPS. Science 307, $1313-1317$.

Edited by: P. C. F. Oyston 